Keywords: Serum metabolomics; Stress metaboIomics; Biomarkers; Direct Infussion Mass Spectrometry.

\title{
Direct infussion Electrospray Mass Spectrometry as a new non-invasive tool for serum metabolomics in induced-stress subjects
}

\author{
Mónica Lorenzo-Tejedora \\ Concepción de la Cámarab \\ Raul Lopez-Anton ${ }^{c}$ \\ Raquel Bailond \\ Jordi Aguilóe \\ María Luisa Bernal Ruiz ${ }^{a, \star}$ \\ a Department of Pharmacology and \\ Physiology. Faculty of Medicine. \\ University of Zaragoza

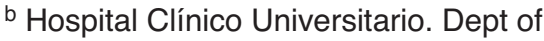 \\ Medicine and Psychiatry. University \\ of Zaragoza \\ ${ }^{c}$ Department of Psychology and Sociology. \\ University of Zaragoza \\ d BSICoS, I3A, University of Zaragoza, \\ IIS Aragón, CIBER-BBN, Zaragoza \\ e Universitat Autònoma de Barcelona, \\ CIBER-BBN, Barcelona
}

SPAIN

\begin{abstract}
Background and Objectives: Nanotechnology is becoming a tool for the study of changes in the metabolome of patients in different states of disease. Analytical techniques such as Electrospray Mass Spectrometry, allow to find biomarkers by determination of metabolites. Nowadays, there is not an objective analytical approach for diagnosis of stress. Thus, the objectives of this pilot work are: Describing the development of a fast, direct and non-invasive analytical protocol, applied for the first time, to study the metabolomic profile of patient s different states through a disease. Testing the protocol in a pilot sample with non-stressed and stress-induced subjects.

Methods: High resolution direct infusion electrospray mass spectrometry has been used to analyse the metabolome of blood samples $(0.3 \mathrm{ml})$ from six subjects.

Results: Data prove a clear discrimination between non-stressed and stressed states in the metabolome. Data showed different predominant metabolites in both states. Results allow objective characterization of the state of the patient.

Conclusions: Although this is a pilot study, the method was successful in discriminating different metabolites in non-stressed and stress-induced subjects.
\end{abstract}




\section{Introduction}

The main aim of biochemical medicine is to understand the molecular biological mechanisms and being able to monitor them ${ }^{1}$. These mechanisms can be studied by analysing the expressed genes and their corresponding proteins ${ }^{2}$. However, our interest lies in knowing the differences in patients' biosynthetic cycle, which can help us to understand the different disease processes in subjects who present equivalent biological and environmental conditions ${ }^{3}$. The human cells produce small molecules known as metabolites. A metabolic profile is important to understand the human biology $y^{4,5}$.

Metabolomics allows fast and simultaneous measurement of hundreds of metabolites of medical interest. Thus it is one of the tools to open up new prospects with great potential in the study of diseases ${ }^{6,7}$ such as coronary artery disease $^{11}$, in diagnostic protocols ${ }^{8}$, the search for biomarkers ${ }^{9}$ and the development of drugs ${ }^{10}$. The metabolomic analysis of cerebrospinal fluid has enabled us to predict the clinical outcome of subarachnoid haemorrhage $^{12}$. Metabolomic profiling in serum has also allowed us to classify patients with neurological diseases such as amyotrophic lateral sclerosis $^{13}$.

Stress affects the biological functions causing biochemical instability and biological failure ${ }^{14,15}$. Stressors can promote disturbances ranging from immune system dysfunction to psychiatric disorders ${ }^{16,17}$, and cause neuropathological changes similar to those provoked by Alzheimer's disease ${ }^{18}$ or dementia ${ }^{19}$.

Stress is considered as a situation of biological and psychological activation triggered by the interaction of the individual with external agents that force his/her capacity of adaptation and survival ${ }^{20}$. Stress is associated primarily with cortisol, the main stress hor- mone which alters the body's functions ${ }^{21,23}$. The biosynthesis of the cortisol cycle has been well-studied ${ }^{24-26}$. Cortisol has wideranging systemic effects on the organism: regulating insulin in blood ${ }^{27}$, linked to the development of type II diabetes disease, to inflammatory processes (tumors) or immunological diseases ${ }^{28}$, obesity, cardiovascular diseases, thyroid disorders, mental illness ${ }^{29}$ and depression ${ }^{30,31}$. The measurement of cortisol does not provide an overview of the biochemical profile, so individual specific metabolomic conditions in each patient cannot be known neither if there is a connection to any other pathology ${ }^{32}$. Likewise, tests carried out by psychologists to diagnose stress are highly subjective. They are composed of questions that the researched subject must understand and also differentiate and compare their emotional state. Besides, they also depend on the therapist's conclusions interpreting the answers and the morphological analysis of the patient ${ }^{33}$.

Subsequently, stress has been considered, in this work, as an important factor to be studied under the metabolomic approach. The metabolome is the response of an organism to an alteration, and is a very useful tool for understanding the biological mechanisms of complex organisms. It is a step beyond the classical approach of biochemistry. This new approach applied to the study of stress is of importance since its method of diagnosis is limited. Nor are we aware of the behaviour of the metabolome in the early stages of any symptoms (stress) or what future links, there may be to any subsequent diseases occurring in the individual. This could allow us to set a specific diagnosis for each case and therefore initiate treatment prior to the development of the disease. Therefore, the identification of key biomarkers with a fast, simple and complete analysis is important for the early detection of certain diseases. In addition, 
since stress is a multifactorial problem, it can be understood that it is unlikely that just a single biomarker (cortisol) is relevant to its possible diagnosis. A panel of biomarkers and their values are likely to be a more sensitive and specific approach to study the disease $\mathrm{e}^{34}$.

Current analytical methods such as NMR and mass spectrometry (MS) are often combined with chromatography, which requires several millilitres of sample and a specific clinical protocol ${ }^{35}$. Chromatography gives good analytical results but, it has limitations when it comes to the yield and performance of the sample. There are attempts to apply new techniques to reduce analysis time and to increase the information obtained from each analy$\operatorname{sis}^{36,37}$ the yield per sample and the reliability.

Recently, analytical science is searching for direct methods injecting samples directly into the mass spectrometer ${ }^{38}$ improving reproducibility for the same non-target metabolites and, increasing responsiveness and yield ${ }^{39}$.

The aim of this pilot study is to use a noninvasive and precise technique as direct infusion mass spectrometry, to find and characterize metabolic differences in two different biological situations of an individual: relaxed and stressed states.

\section{Materials and methods}

\section{Participants and sampling}

This study has been run with volunteer subjects from the University population. The subjects had an age range from 18 to 30 years (both gender). Before performing the sessions for induced-stress and relaxation, each volunteer read and completed a survey with a questionnaire which was focused on: on the volunteer's habits and life style, consumption of coffee, alcohol, psychotropic substances, and smoking habits. Questions in relation to chronic diseases or prescribed regular drug intake were included. Possible depressed patients with pre-existing physical or mental disorders, medication, and/or illicit drug use, were excluded from the group after this personal evaluation.

Individuals were adequately informed, and they gave their informed consent to participate in the study which was approved by the Ethics Committee at Clinical Hospital "Lozano Blesa" of the University of Zaragoza, Spain.

Demographic data of the samples that were selected are shown in Table 1.

Table 1

Demographic data of selected samples for the study.

\begin{tabular}{lcccc} 
Sample number & Age (years) & Weight $(\mathrm{kg})$ & Height $(\mathrm{cm})$ & Gender \\
\hline Sample 1 & 20 & 67 & 171 & Male \\
\hline Sample 2 & 19 & 78 & 180 & Male \\
\hline Sample 3 & 19 & 77 & 185 & Male \\
\hline Sample 4 & 19 & 54 & 162 & Female \\
\hline Sample 5 & 22 & 93 & 182 & Male \\
\hline Sample 6 & 24 & 68 & 176 & Male \\
\hline Sample 7 & 21 & 61 & 170 & Female \\
\hline Sample 8 & 20 & 70 & 175 & Male
\end{tabular}




\section{Design of the relaxation and stress sessions}

The design and preparation of the sessions were performed under the framework of the "ES3 project" 40 . Data acquisition and sample preparation were done by the Zarademp team in collaboration with BSICoS I3A team. Each research subject underwent, a 35-minute relaxation session including audio and guided relaxation, in a space with dimmed lights while in a comfortable position. This state is considered in this work as basal. Stress was induced by a modification of the Trier Social Stress Test, which is widely used in stress research $^{41,42}$. The protocol follows the one described in reference ${ }^{43}$.

The State Trait Anxiety Inventory (STAI) ${ }^{44}$, Spanish version ${ }^{45,46}$, measures both state anxiety and trait anxiety; The STAI has demonstrated adequate reliability (Cronbach's alpha 0.90 to 0.94 for trait anxiety and state anxiety, respectively) ${ }^{45,46}$.

The Perceived Stress Scale (EEP) is the most widely used psychological instrument for measuring the perception of stress. This Likert-type scale measures a person's perception of potentially stressful events ${ }^{47}$. The Spanish version ${ }^{48}$ of the EEP demonstrated adequate reliability (internal consistency, alpha $=0.81$, and test-re-test $r=0.73$ ) validity (concurrent) and sensitivity. Additional data indicate adequate reliability (alpha $=0.82$, test-retest, $r=0.77$ ), validity and sensitivity of the short version of 10 items which is used in this study.

The stress sessions began after a prior 10minute relaxation session. To stress the research subjects a protocol based on an emotional stress test was used. Biophysical parameters and biological sampling were carried out once the session had ended and were recorded throughout the entire test. Biochemical parameters and blood pressure were taken at the end of each session (Table 2). The biological samples were stored in sterile, airtight compartments at $-80^{\circ} \mathrm{C}$ until the time of analysis.

\section{Reagents and samples}

Blood samples for analysis were taken by pricking participants' fingers. Approximately $0.3 \mathrm{ml}$ was collected in a sterilized container tube with no chelating agent ethylene diamine tetraacetic acid (EDTA). The samples were immediately protected from light and stored at $-80^{\circ} \mathrm{C}$ until analysis. No blood sample underwent any pre-treatment prior to mass spectrometry analysis.

Mass spectrometry (LC-MS) grade methanol and water for ESI analysis were used (Fisher). Formic acid (99\% purity- Fluka) was applied as a protonate agent. $0.45 \mu \mathrm{m}$ PVDF sterile filters 100 PC2 ROTH (Carl Roth GmbH.CoKG- TECNOCROMA) were used.

\section{Sample preparation}

Samples were thawed on ice and homogenized with vortex agitation. $100 \mu$ l of unclothed blood was taken and $100 \mu \mathrm{l}$ of water was added and homogenized. $100 \mu \mathrm{l}$ of the solution was collected and added to $300 \mu$ of LC-MS grade methanol. The resulting solution was homogenized and left to stand for 30 minutes at $-20^{\circ} \mathrm{C}$. Subsequently, samples were centrifuged at $10,000 \mathrm{rpm}$ for 10 minutes at $4^{\circ} \mathrm{C}$ and the supernatant filtered with a sterile PVDF $0.45 \mu \mathrm{m}$ filter avoiding the dead volume. For positive mode detection, LC-MS grade methanol containing $0.1 \%$ formic acid (99\% purity) was added to the sample, obtaining a sample dilution of 1:1000. 
Table 2

Physiological measurements in the relalaxation-stress sessions.

\begin{tabular}{|c|c|c|}
\hline Biophysical variables & Location on the body & Measurement features \\
\hline Skin temperature (ST) & $\begin{array}{l}\text { Little finger of non-dominant } \\
\text { hand and cheek }\end{array}$ & Continuous $(250 \mathrm{~Hz})$ \\
\hline Skin conductance (SC) & $\begin{array}{l}\text { First phalanx of ring and index fingers, } \\
\text { non-dominant hand }\end{array}$ & Continuous $(250 \mathrm{~Hz})$ \\
\hline Electrocardiogram (ECG) & (3 sources) thorax & Continuous $(1 \mathrm{KHz})$ \\
\hline Respiratory signal (RR) & Thorax & Continuous $(250 \mathrm{~Hz})$ \\
\hline Blood pressure (BP) & Arm & $\begin{array}{l}\text { Non-continuous } \\
\text { (at the beginning and end } \\
\text { of each session) }\end{array}$ \\
\hline Wave pulse (PPG) & Middle finger of non-dominant hand & Continuous $(250 \mathrm{~Hz})$ \\
\hline Electromyogram (EMG) & Left trapezium and orbicular muscles & Continuous $(1 \mathrm{KHz})$ \\
\hline Biochemical variables & Location & Measurement features \\
\hline Prolactin & Blood & At the end of session (10:45) \\
\hline \multicolumn{3}{|l|}{ Copeptin } \\
\hline \multicolumn{3}{|l|}{ Glucose } \\
\hline Cortisol & Saliva & $\begin{array}{l}10 \text { minutes following relaxation } \\
(10: 20) \text { and at the end of the } \\
\text { session }(10: 45)\end{array}$ \\
\hline
\end{tabular}

\section{Instrumentation}

Measurements were taken using a hybrid triple quadruple/linear ion trap mass spectrometer 4000 QTRAP LC/MS/MS System (AB Sciex.) with an electrospray ionization (ESI) source interface for high-sensitivity, fullscan MS and MS/MS spectra with high selectivity. The system was operated using Analyst software version 1.5.2 (Build 5704) (AB Sciex) for data acquisition and pre-processing.

For MS/MS analysis collision-induced dissociation (CID) mode was used and was set to $30 \%$ to $50 \%$ normalized collision energy (CE) for selected molecular Mass/Charge peaks.

\section{Data Processing and compound identification}

Data was processed by using the Marker View 1.2 software (AB SCIEX) for statistical analysis, by picking and matching $\mathrm{m} / \mathrm{z}$ across samples resulting into a two dimensional matrix of peaks and intensities after data normalization. $\mathrm{m} / \mathrm{z}$ tolerance equalled to $50 \mathrm{ppm}$ and presence of each feature in at least 3 samples. After performing a $T$-test, the subsequence principal component analysis Pareto (PCA) was performed. Metabolites with $p$-value lower than 0.05 were considered statistically significant. In order to find differences of biomarker concentration between stressed and relaxed groups Variable Importance in Projection (VIP) was used for pre- 
dominant $m / z$ selection in each group of data. $R^{2}$ and $Q^{2}$ values provided good quality of the resulting model.

Identification of significant compounds was made manually matching the experimental accurate mass and spectra with available information contained in metabolic databases. By means of MS/MS analysis, metabolite structure was confirmed by characteristic fragments described in literature or compared to standard compounds.

\section{Results}

Only the hydrophilic portion of the metabolome is presented in this paper, sample profiles were acquired for each research subject in relaxed (basal) and stress-induced condition (Figure 1). Each of these profiles showed a large number of signals, around $1500 \mathrm{~m} / \mathrm{z}^{49}$. Each spectrum can be considered a metabolomic fingerprint of the subject in each state. The matrix effect on metabolomic profiles is a significant factor and is taken into account in the study. It was attenuated by optimizing the dilution factor, so the intensity of the $\mathrm{m} / \mathrm{z}$ signal of each metabolite has a linear relationship with the concentration when dilutions are high ${ }^{50,51}$.

In order to compare profiles of subjects in both states, two groups of $\mathrm{m} / \mathrm{z}$ signal/intensity were established (X-B for basal-non-stressed state, X-E for induced-stress state). A chemometric partial least squares discriminant (PLS-DA) analysis was performed using the $\mathrm{m} / \mathrm{z}$ signal/intensities of the samples from the two groups ${ }^{52}$. The models gave rise to a good classification outcome as shown in the score plots (Figure 2). These groups of results were evaluated statistically and the resulting models showed values in line with quality para- meters $R^{2}$ and $Q^{2}$ (explained variance of approximately $99 \%$ and a predicted variance above $50 \%)^{53}$. T-test analysis with $p$-values and data modelling using the PLS (Principal Least Squares) progression were carried out ${ }^{54,55}$. A large number of signals could be studied in the discrimination of classes considering the Variable-Importance-in-Projection-(VIP) which was set at a minimum value of 2. Generally, VIP set at 1 can be considered important in a given model ${ }^{56}$. With regard to the reproducibility of the method, this could be considered good with variation coefficients $(C V \%)$ below $20 \%$ for the metabolites identified. Thus, subjectivity in the selection process is avoided ${ }^{57}$.

A complex metabolite profile was obtained since there has not been any pre-treatment in the process of analysis. Around 1500 of MS peaks out of a sample were detected (Figure 1). All peaks were analyzed, evaluated and exported for statistical analysis. In order to differentiate between subjects in a basal and stress-induced state, the normalized data was classified into two sets (basal and stressed), and evaluated by $T$-test and partial least squares discriminant analysis-PCA (Figure 2).

Statistic results are shown in Table 3 . Model built with the data, provided a good classification in two groups. Thus, by PCA we could confirm that the observed behavior is real and not random and study $m / z$ peaks that can be specific for one group.

The more discriminant signals were selected according to Variable-Importance-inthe-Projection-(VIP) for later study by MS/ MS and molecule identification (Table 3). After the data processing, we selected $\mathrm{m} / \mathrm{z}$ values that presented a $p$-value which denoted a correct statistical hypothesis. Many low molecular weight metabolites presented alterations in stressed state samples as compared to basal state samples. 
a)

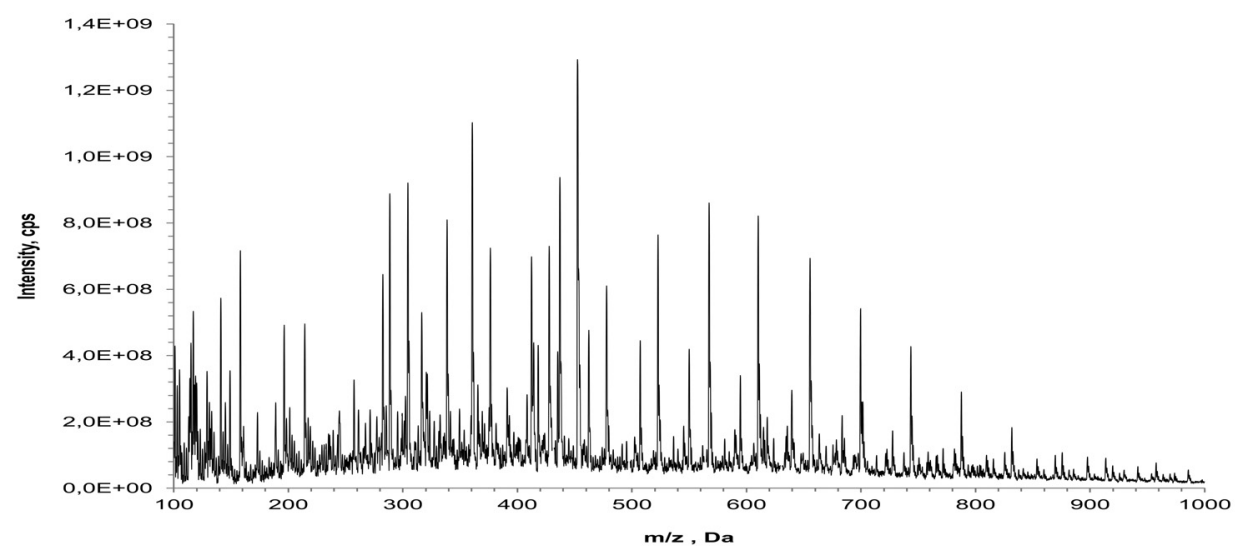

b)

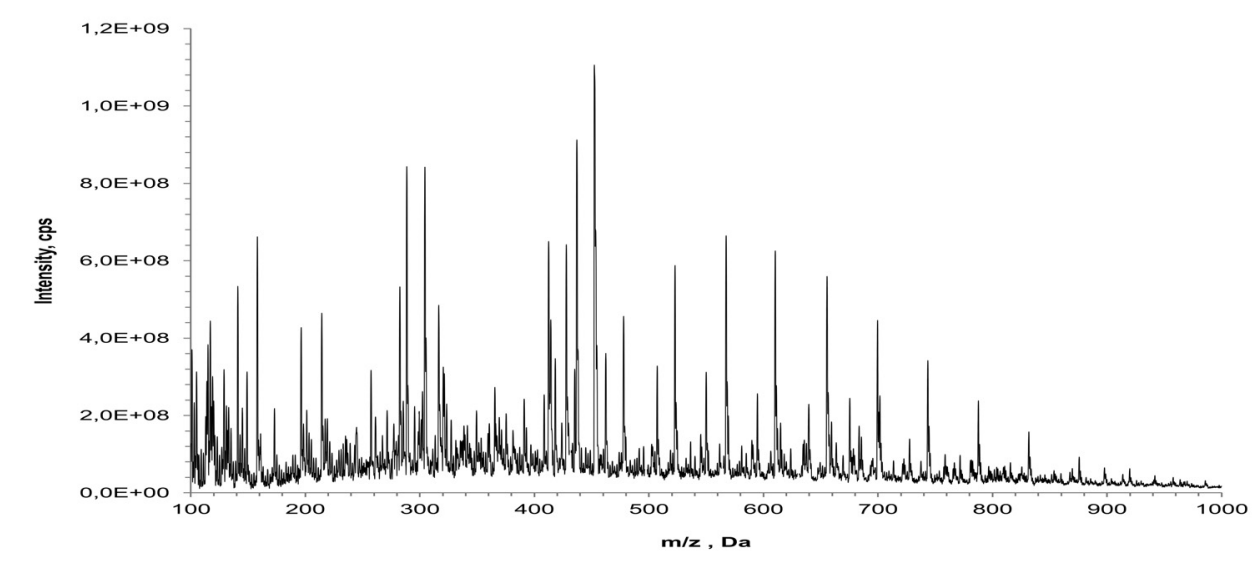

Figure 1. Example of full spectra metabolome profile of a subject detected for Basal (non-stressed) and stress-induced states.

(Range m/z (mass/charge): 100 to 1000). a) Basal state subject, b) stress-induced state subject.

More than 1500 signals ( $\mathrm{m} / \mathrm{z}$ for possible compounds) were detected for each sample in each group of subjects.

In order to distinguish prevailing metabolites in both basal and stress groups, peaks with specific $p$-value and good CV where evaluated considering the VIP. Together with their VIP value, the PCA statistical analysis clarifies if the metabolite is of greater importance in each group (Table 3).
Examples of specific molecules for stressed subjects are the peak $m / z 363.4653[\mathrm{M}+\mathrm{H}]^{+}$, $(-7.26 \Delta \mathrm{ppm})($ Table 3$)$ which was identified as Cortisol/Hydrocortisone and Estrone $\mathrm{m} / \mathrm{z}$ $271.1706[\mathrm{M}+\mathrm{H}]^{+}$. Following this same procedure other compounds from steroid hormone metabolism were detected. Similarly, 


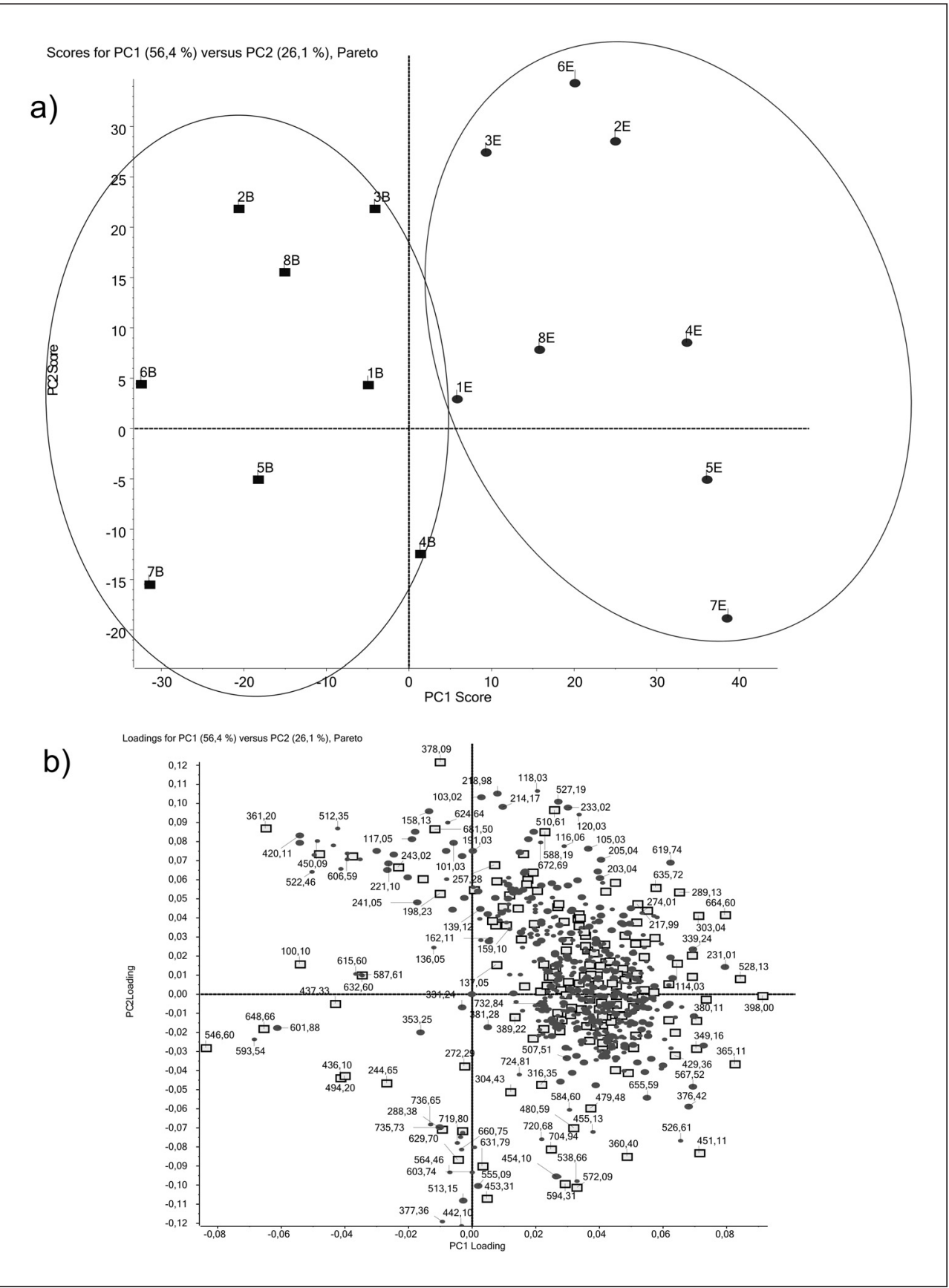

Figure 2. Diagrams of the statistical results of the whole MS (Mass Spectrometry) data found in both groups of subject states after using bioinformatics:

a) Scores plots of Pareto PCA for basal state subject (squares), stress-induced state subjects (circles).

b) Pareto loadings for PCA $\mathrm{m} / \mathrm{z}$ (molecular mass/charge) peaks. 


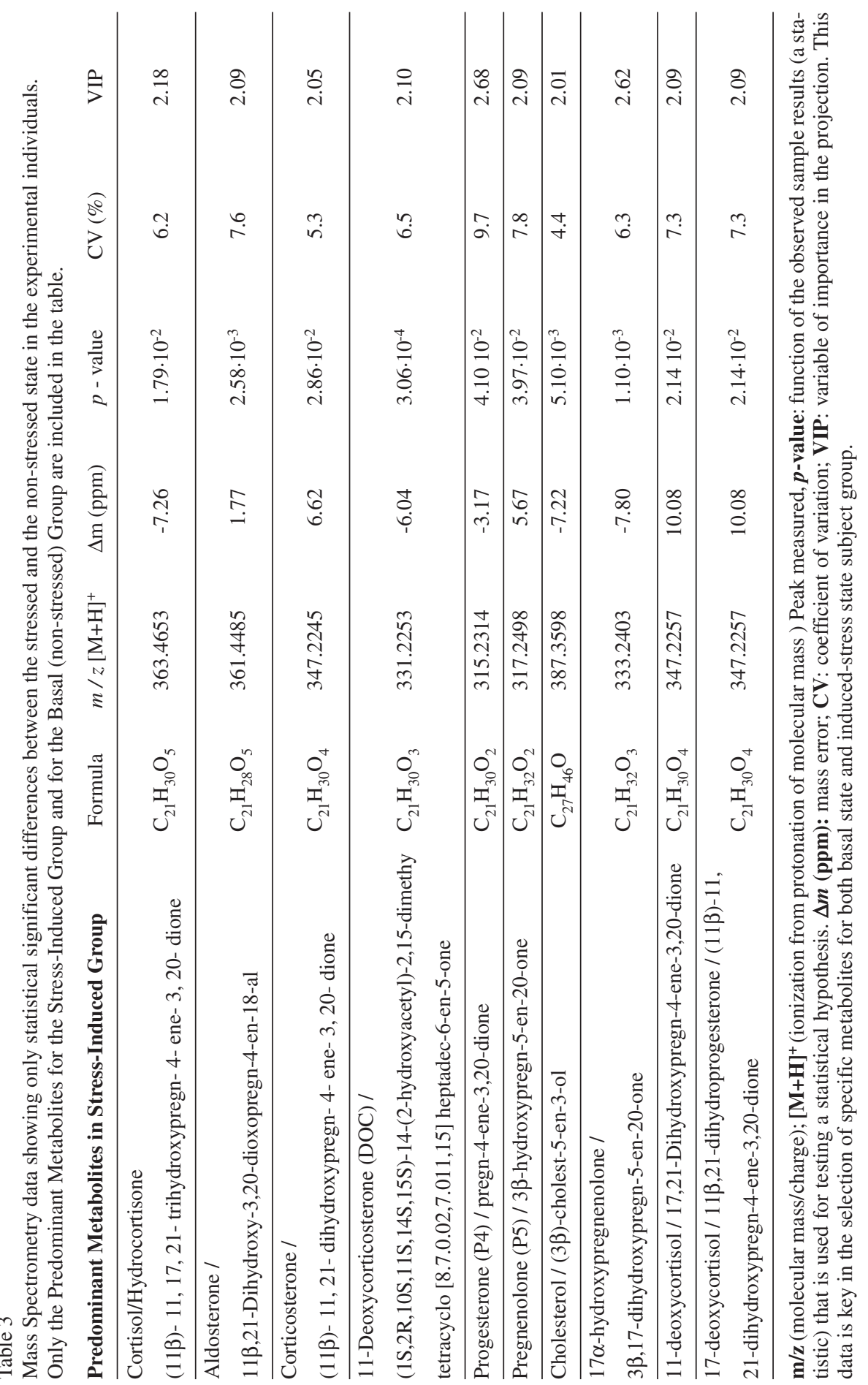


MÓNICA LORENZO-TEJEDOR ET AL.

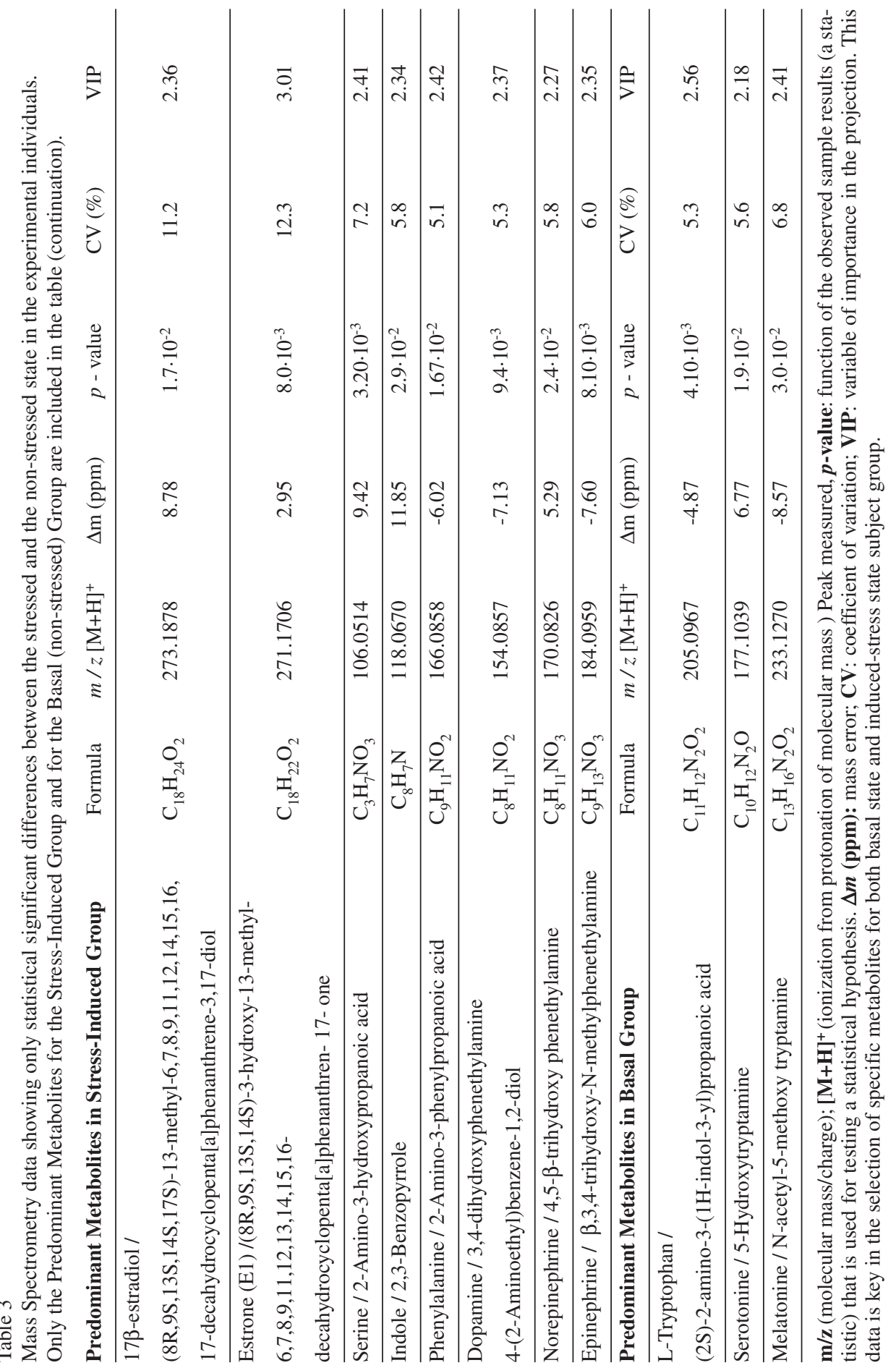


statistical values expressed specificity for other metabolites in the metabolism of Serotonine $\left(\mathrm{m} / z\right.$ 177.1039 $\left.[\mathrm{M}+\mathrm{H}]^{+}\right)$, such as L-Tryptophan $\left(\mathrm{m} / \mathrm{z} 205.0967[\mathrm{M}+\mathrm{H}]^{+}\right)$or Melatonine $\left(\mathrm{m} / z, 233.1270[\mathrm{M}+\mathrm{H}]^{+}\right)$, which were predominant in samples of basal state (Table 3).

MS/MS analysis was performed to confirm elemental composition of the peaks and identify the studied biomarkers (Table 4).

\section{Discussion}

This pilot study was designed to use direct infusion mass spectrometry as a non-invasive and precise technique to perform a metabolic-profiling screening and characterized metabolic differences in relaxed and stressedinduced subjects.

The comparison of the metabolomic composition profiles of the different states of the subject will form the basis of the search for biochemical data. The results show that the characterized metabolites by means of the new technique applied in this pilot work were different in the two compared states; relaxed and stressed-induced situations. The cortisol and their related metabolites are predominant in the stress state while serotonine and melatonine, among other metabolites, were found to be predominant in the relaxed state when comparing both groups of metabolicprofiling data.

Through studies in the past we know significantly more about the neurobiological correlations of stress ${ }^{58}$. What we do not know is why these changes are associated with the development of disorders in some people but not in others. The importance of understanding the individual differences is key to the whole comprehension of stress.

Therefore, the possibility of developing a non-invasive, direct and fast method of analy- sis, to assist the study of stress in an objective manner, can be considered a step forward both patient correct diagnosis and treatment Moreover, given the risk that a patient will develop a neurological disease of greater medical significance, it would be very valuable to have, a prior basal and stressed state metabolomic profile to assist professionals treating said patient.

Since the complexity of the human metabolome is of a great magnitude, different complementary techniques for its study are being developed ${ }^{59-61}$. The techniques most commonly used have been those based on $\mathrm{NMR}^{34,62-64}$. However, it has its drawbacks since it cannot detect metabolites in very low concentrations, it requires blood samples in millilitres and a protocol that is slow and costly.

Therefore, the application of nanotechnology techniques in search of a non-invasive, fast and direct analytical method that could offer a patient's metabolomic-profile at a specific time is needed. In the method that we have developed the required sample amount is in microlitres (one drops of blood from the subject's fingers is enough). The sample does not undergo pre-treatment which might adulterate or remove biochemical data, so a more complete metabolomic fingerprint is expected.

Many authors in psychiatric or neurological disease research use metabolic-profiling associated to these diseases including LC/ MS, GC/MS and EC/MS ${ }^{60,65,66}$. However direct infusion mass spectrometry used in this work is completely unexplored.

This tool provides for the possibility of an analysis of multiple metabolites in a simple run, obtaining a metabolomic-fingerprint. Furthermore, the low-time-consuming step before MS allows for a faster analysis of samples, which reduces instrumental work and drift between analysis, increasing reproducibility and improving accuracy ${ }^{67}$. This means an im- 


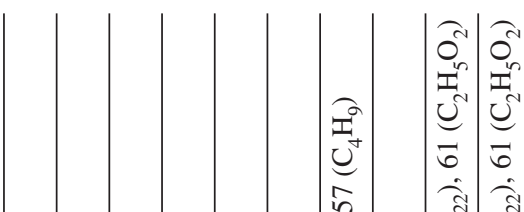

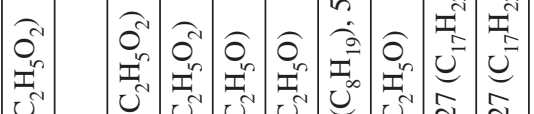

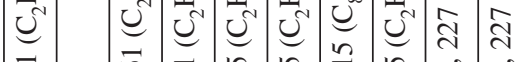

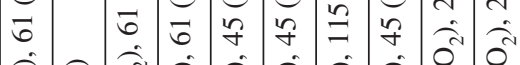

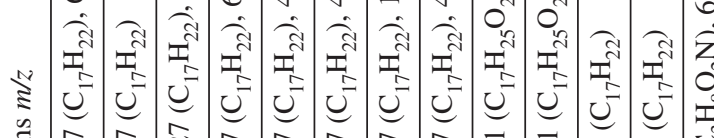

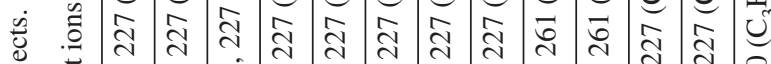

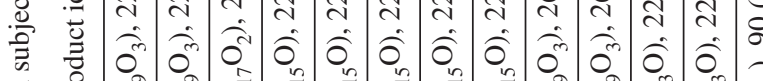

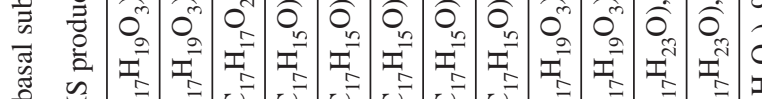

可

空

西

:

言

离

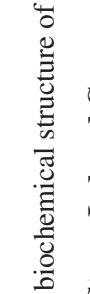

$\because$

竞

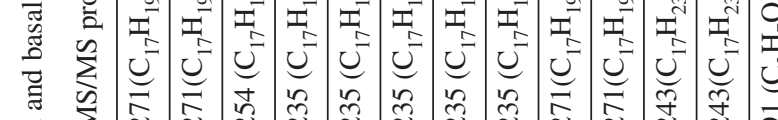

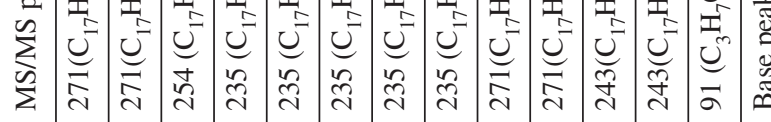
穴 =

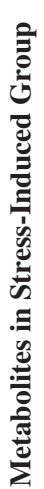

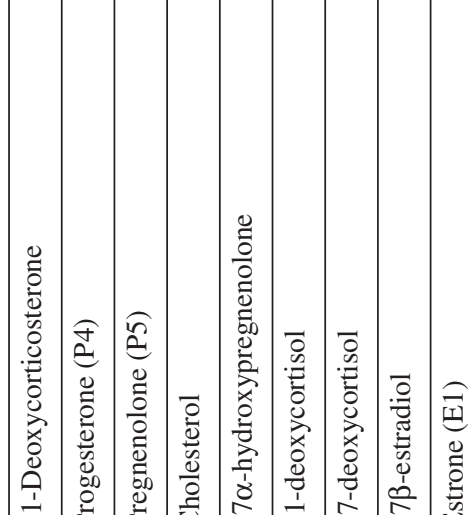

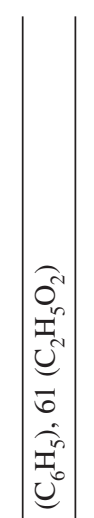

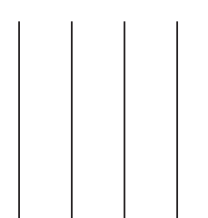

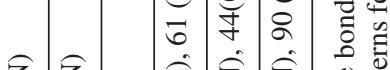

z)

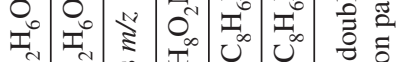
ป \&

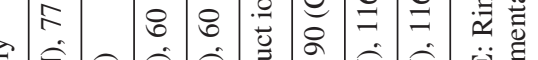

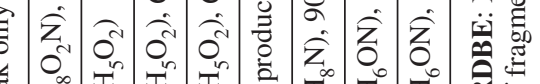
年

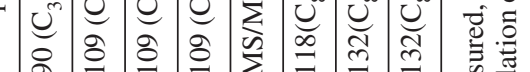

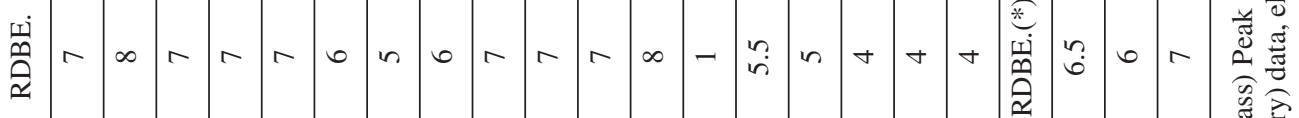

至

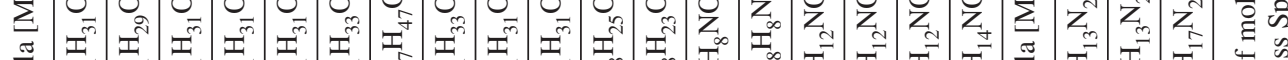

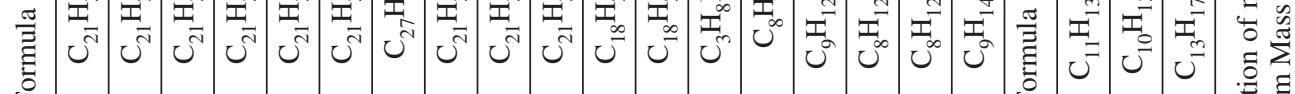

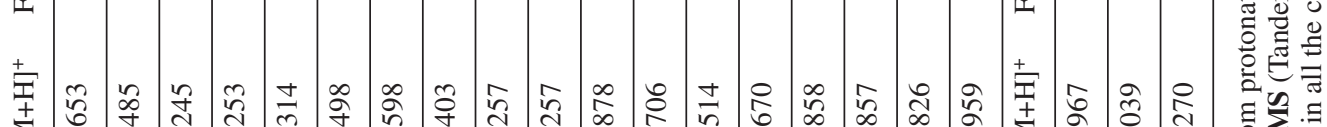

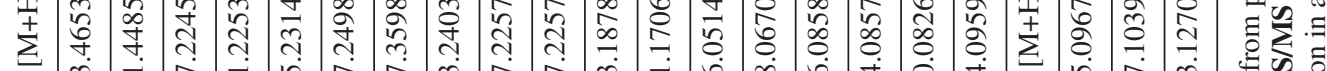

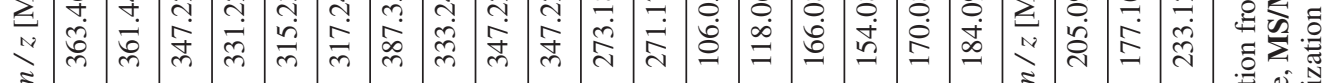

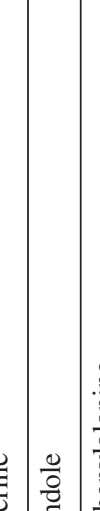
$\lesssim$

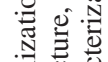

tin

高垔高 
provement in metabolomics applied to biological samples as previously described.

Since there are no published papers similar to this proposed-one, statistical models whose quality would be comparable or higher than other studies of psychiatric or neurological disorders have been used ${ }^{27,39}$.

Data Information Management Systems (Bioinformatics), which has been already used in similar approaches in metabolomics applied to diseases ${ }^{10,11,12,27,39}$, allowed a faster and more effective filtering and selection of data.

Mass spectrometry which will enable a more accurate measurement of mass and a better structural elucidation of the compounds to be studied using MS/MS was selected ${ }^{68,69}$. Direct infusion mass spectrometry can be considered an effective tool in the study of metabolomic anomalies in stress research as proposed in this paper, since it has been successful in other diseases or pathologies ${ }^{70,71}$. Direct injection analysis into the ionization source of the mass spectrometer without prior chromatographic separation is a technique commonly used with atmospheric pressure ionization techniques (ESI). It is a high-throughput approach already used in metabolicfingerprinting in crude fungal extracts ${ }^{58}$ and yeast intracellular metabolomics ${ }^{72}$. There is not yet any study done in the study of stress. However, the results prove that the methology applied is robust.

Since the predominant metabolites are different between the two studied states, this allows to objectively characterizing in which state is any of the subjects.

According to Cowen P.J. ${ }^{58}$ and other authors ${ }^{72-74}$ our results confirm that serotonin and related metabolites decrease in stress situation. While during stress induction, glucocorticoid secretion increases as a response to stress ${ }^{58,73-76}$ becoming predominant.
Therefore, an objective tool, as the presented protocol, could provide important information to aid in this aim and, although the presented results emerged from a pilot trial, they seem promising.

Though the specificity and sensibility of Mass Spectrometry techniques for low-volumes and low-concentration samples have been already demonstrated, there is yet the need to address the problem of false negatives/positives when compared with the psychometric evaluation. Further research is currently being done to complete the evaluation of this technique.

\section{Conclusion}

The described method, based in this pilot study, is presented as a tool to identify biomarkers in stress-induced subjects. Direct Infusion Mass Spectrometry protocol in this work has been adapted to be applied for the first time in the classification stress-induced subjects. The results provide a preview of preliminary measurable outcomes of the initial objectives discriminating among stressed or relaxed subjects. It opens the possibility to compare metabolomic profiles. Furthermore, this methodology is a quick, direct and noninvasive method for patients. Nevertheless, further work needs to be done, such as, accurate quantification of compounds. On the other hand, a more exhaustive and complete research should be developed to be able to have a full screening of results among different types of patients and a comparative analysis between metabolomic/biological data and psychological diagnose. However, we consider that it can be foreseen many applications for the proposed protocol such as quick-quality-diagnose, pre-treatment of side effects, therapeutic target search etc., which can be of interest in the present-day analytical field. 


\section{Conflict of interest}

The authors declare that they have no competing interests.

\section{References}

1. Richard AH, Denise RF. Biochemistry. First edition ed.: Lippincott Williams \& Wilkins; 2011.

2. Tuller T, Atar S, Ruppin E, Gurevich M, Achiron A. Common and specific signatures of gene expression and protein-protein interactions in autoimmune diseases. Genes Immun. 2013; 14(2): 67-82.

3. Madsen R, Lundstedt T, Trygg J. Chemometrics in metabolomics-a review in human disease diagnosis. Anal Chim Acta. 2010; 659(1-2): 23-33.

4. Medina S, Dominguez-Perles R, Gil JI, Ferreres F, GilIzquierdo A. Metabolomics and the diagnosis of human diseases-a guide to the markers and pathophysiological pathways affected. Curr Med Chem. 2014; 21(7): 823-848.

5. Mamas M, Dunn WB, Neyses L, Goodacre R. The role of metabolites and metabolomics in clinically applicable biomarkers of disease. Arch Toxicol. 2011; 85(1): 5-17.

6. Lv H, Hung CS, Chaturvedi KS, Hooton TM, Henderson JP. Development of an integrated metabolomic profiling approach for infectious diseases research. Analyst. 2011; 136(22): 4752-63.

7. Xiao JF, Varghese RS, Zhou B, Nezami Ranjbar MR, Zhao Y, Tsai TH, et al. LC-MS based serum metabolomics for identification of hepatocellular carcinoma biomarkers in Egyptian cohort. J Proteome Res. 2012; 11(12): 5914-23.

8. Zheng P, Gao HC, Li Q, Shao WH, Zhang ML, Cheng $\mathrm{K}$, et al. Plasma metabonomics as a novel diagnostic approach for major depressive disorder. J Proteome Res. 2012; 11(3): 1741-8.

9. Peng B, Li H, Peng XX. Functional metabolomics: from biomarker discovery to metabolome reprogramming. Protein Cell. 2015; 6(9): 628-37.

10. Shah NJ, Sureshkumar S, Shewade DG. Metabolomics: A Tool Ahead for Understanding Molecular Mechanisms of Drugs and Diseases. Indian J Clin Biochem. 2015; 30(3): 247-54.

11. Brindle JT, Antti H, Holmes E, Tranter G, Nicholson JK, Bethell HW, et al. Rapid and noninvasive diagnosis of the presence and severity of coronary heart disease using $1 \mathrm{H}-$ NMR-based metabonomics. Nat Med. 2002; 8(12): 1439-44.
12. Dunne VG, Bhattachayya S, Besser M, Rae C, Griffin JL. Metabolites from cerebrospinal fluid in aneurysmal subarachnoid haemorrhage correlate with vasospasm and clinical outcome: a pattern-recognition $1 \mathrm{H}$ NMR study. NMR Biomed. 2005; 18(1): 24-33.

13. Kaddurah-Daouk RC, Beecher BS, Kristal WR, Matson M, Bogdanov DA. Bioanalytical Advances for Metabolomics and Metabolic Profiling. Pharmagenomics 2004; 4: 46-52.

14. Cohen S, Janicki-Deverts D, Miller GE. PSychological stress and disease. JAMA. 2007; 298(14): 1685-7.

15. Maurer CW, LaFaver K, Ameli R, Toledo R, Hallett M. A biological measure of stress levels in patients with functional movement disorders. Parkinsonism Relat Disord. $2015 ; 21(9)$ : 1072-5.

16. Duman EA, Canli T. Influence of life stress, 5-HTTLPR genotype, and SLC6A4 methylation on gene expression and stress response in healthy Caucasian males. Biol Mood Anxiety Disord. 2015; 5: 2. eCollection 2015.

17. Generaal E, Vogelzangs N, Macfarlane GJ, Geenen R, Smit JH, de Geus EJ, et al. Biological stress systems, adverse life events and the onset of chronic multisite musculoskeletal pain: a 6-year cohort study. Ann Rheum Dis. 2015 Apr 22.

18. Sotiropoulos I, Catania C, Pinto LG, Silva R, Pollerberg GE, Takashima A, et al. Stress acts cumulatively to precipitate Alzheimer's disease-like tau pathology and cognitive deficits. J Neurosci. 2011; 31(21): 7840-7.

19. Lu T, Aron L, Zullo J, Pan Y, Kim H, Chen Y, et al. REST and stress resistance in ageing and Alzheimer's disease. Nature. 2014; 507(7493): 448-54.

20. Grippo AJ, Scotti MAL. Stress and Neuroinflammation. In: Inflammation in Psychiatry. Halaris A and Leonard BE (Eds). Basel, Karger, 2013, vol 28, pp 20-32.

21. Dedovic K, Ngiam J. The cortisol awakening response and major depression: examining the evidence. Neuropsychiatr Dis Treat. 2015; 11: 1181-9. eCollection 2015. Review.

22. Hansson PB, Murison R, Lund A, Hammar A. Cognitive functioning and cortisol profiles in first episode major depression. Scand J Psychol. 2015; 56(4):379-83.

23. Shimizu A, Nishiumi H, Okumura Y, Watanabe K. Depressive symptoms and changes in physiological and social factors 1 week to 4 months postpartum in Japan. J Affect Disord. 2015; 179: 175-82.

24. Belsky J, Ruttle PL, Boyce WT, Armstrong JM, Essex MJ. Early adversity, elevated stress physiology, accelerated sexual maturation, and poor health in females. Dev Psychol. 2015; 51(6): 816-22. 
25. Booij SH, Bos EH, Bouwmans ME, van Faassen M, Kema IP, Oldehinkel AJ, et al. Cortisol and alpha-Amylase Secretion Patterns between and within Depressed and NonDepressed Individuals. PLoS One. 2015; 10(7): e0131002.

26. Rausch J, Gabel A, Nagy K, Kleindienst N, Herpertz SC, Bertsch K. Increased testosterone levels and cortisol awakening responses in patients with borderline personality disorder: gender and trait aggressiveness matter. Psychoneuroendocrinology. 2015; 55: 116-27.

27. Tang J, Tavintharan S, Sum C, Lim S, Ong H. 4b.10: 24 Hour Urine Free Cortisol to Cortisone Ratio is a Novel Biomarker for Increased Left Ventricular Mass in Diabetic Hypertensives. J Hypertens. 2015; 33 Suppl 1: e55.

28. Boehringer A, Tost H, Haddad L, Lederbogen F, Wust S, Schwarz E, et al. Neural Correlates of the Cortisol Awakening Response in Humans. Neuropsychopharmacology. 2015; 40(9): 2278-85.

29. Du X, Pang TY. Is Dysregulation of the HPA-Axis a Core Pathophysiology Mediating Co-Morbid Depression in Neurodegenerative Diseases? Front Psychiatry. 2015; 6: 32.

30. Ahn S, Corwin EJ. The association between breastfeeding, the stress response, inflammation, and postpartum depression during the postpartum period: Prospective cohort study. Int J Nurs Stud. 2015; 52(10): 1582-90.

31. Martocchia A, Stefanelli M, Falaschi GM, Toussan L, Ferri C, Falaschi P. Recent advances in the role of cortisol and metabolic syndrome in age-related degenerative diseases. Aging Clin Exp Res. 2015.

32. Wester VL, van Rossum EF. Clinical applications of cortisol measurements in hair. Eur J Endocrinol. 2015; 173(4): M1-10.

33. Thompson DJ, Weissbecker I, Cash E, Simpson DM, Daup M, Sephton SE. Stress and cortisol in disaster evacuees: an exploratory study on associations with social protective factors. Appl Psychophysiol Biofeedback. 2015; 40(1): 33-44.

34. Shi B, Tian J, Xiang H, Guo X, Zhang L, Du G, et al. A (1)H-NMR plasma metabonomic study of acute and chronic stress models of depression in rats. Behav Brain Res. 2013; 241: 86-91.

35. Wu K, Li W, Song J, Li T. Production, Purification, and Identification of Cholest-4-en-3-one Produced by Cholesterol Oxidase from Rhodococcus sp. in Aqueous/Organic Biphasic System. Biochem Insights. 2015; 8(Suppl 1): 1-8.

36. Kuehnbaum NL, Gillen JB, Kormendi A, Lam KP, DiBattista A, Gibala MJ, et al. Multiplexed separations for biomarker discovery in metabolomics: Elucidating adaptive responses to exercise training. Electrophoresis. 2015.
37. Kuehnbaum NL, Kormendi A, Britz-McKibbin P. Multisegment injection-capillary electrophoresis-mass spectrometry: a high-throughput platform for metabolomics with high data fidelity. Anal Chem. 2013; 85(22): 10664-9.

38. Wood PL. Mass spectrometry strategies for clinical metabolomics and lipidomics in psychiatry, neurology, and neuro-oncology. Neuropsychopharmacology. 2014; 39(1): 24-33.

39. Gonzalez-Dominguez R, Garcia-Barrera T, Vitorica J, Gomez-Ariza JL. Application of metabolomics based on direct mass spectrometry analysis for the elucidation of altered metabolic pathways in serum from the APP/PS1 transgenic model of Alzheimer's disease. J Pharm Biomed Anal. 2015; 107: 378-85.

40. Aguilo J, Ferrer-Salvans P, Garcia-Rozo A, Caja G, Arza A, \& Garzon JM, et al. The ES3 project:Towards a quantitative assessment of stress level. (Acepted), Rev of Neurology. 2015.

41. Jönsson P, Wallergård M, Osterberg K, Hansen AM, Johansson G and Karlson B. 2010. Cardiovascular and cortisol reactivity and habituation to a virtual reality version of the Trier Social Stress Test: a pilot study. Psychoneuroendocrinology. Vol. 35, no. 9, pp. 1397-403.

42. Hellhammer J and Schubert M. 2012. The physiological response to Trier Social Stress Test relates to subjective measures of stress during but not before or after the test. Psychoneuroendocrinology. vol. 37, no. 1, pp. 119-124.

43. Arza A, Garzón JM, Hernando A, Aguiló J, Bailón R. 2015. Towards an Objective Measurement of Emotional Stress: Preliminary Analysis Based on Heart Rate Variability. Proceedings of the 37th Annual International Conference of the IEEE Engineering in Medicine and Biology Society, Milano (Italy), 25-29 August.

44. Spielberger CD, Gorsuch R y Lushene R. Manual for the StateTrait Anxiety Inventory. Palo Alto, California: Consulting Psychologist Press. 1970.

45. Guillén-Riquelme A, Buela-Casal G. Actualización psicométrica y funcionamiento diferencial de los ítems en el State Trait Anxiety Inventory (STAI). Psicothema. 2011; 23(3): 510-15.

46. Aguiló J, Cambra FJ, Ferrer-Salvans P, López-Anton R, Arza A, Valdés, Garzón Rey JM., García-Rozo A, Bailón R, Gonzalez-Marcos A, Armario A, Caja G, Corbí A, Aguiló S. 2015. Proyecto ES3: Intentando la cuantificación y medida del nivel de estrés. Revista de Neurología. "In Press".

47. Cohen S, Kamarck T, Mermelstein R. A global measure of perceived stress. Journal of health and social behavior. $1983 ; 24$ : 385-96. 
48. Remor E. Psychometric properties of a European Spanish version of the Perceived Stress Scale (PSS). Span J Psychol. 2006; 9(1): 86-93.

49. James EL, Parkinson EK. Serum metabolomics in animal models and human disease. Curr Opin Clin Nutr Metab Care 2015; 18(5): 478-83.

50. Beckmann M, Parker D, Enot DP, Duval E, Draper J. High-throughput, nontargeted metabolite fingerprinting using nominal mass flow injection electrospray mass spectrometry. Nat Protoc. 2008; 3(3): 486-504.

51. Niesser M, Koletzko B, Peissner W. Determination of creatinine in human urine with flow injection tandem mass spectrometry. Ann Nutr Metab. 2012; 61(4): 314-321.

52. Mehmood T, Liland KH, Snipen L, Sæbø S. A review of variable selection methods in Partial Least Squares Regression. Chemometrics and Intelligent Laboratory Systems. 2012; 118: 62-9.

53. Sugimoto M, Kawakami M, Robert M, Soga T, Tomita M. Bioinformatics Tools for Mass Spectroscopy-Based Metabolomic Data Processing and Analysis. Curr Bioinform. 2012; 7(1): 96-108.

54. Szyma Å, Ska E, Saccenti E, Smilde AK, Westerhuis JA. Double-check: validation of diagnostic statistics for PLS-DA models in metabolomics studies. Metabolomics. 2011; 8: 3-16.

55. Rajalahti T, Kvalheim OM. Multivariate data analysis in pharmaceutics: a tutorial review. Int J Pharm. 2011; 417(1-2): 280-90.

56. Gromski PS, Muhamadali H, Ellis DI, Xu Y, Correa E, Turner ML, et al. A tutorial review: Metabolomics and partial least squares-discriminant analysis - a marriage of convenience or a shotgun wedding. Anal Chim Acta. 2015; 879: 10-23.

57. Kumar C, Mann M. Bioinformatics analysis of mass spectrometry-based proteomics data sets. FEBS Lett. 2009; 583(11): 1703-12.

58. Smedsgaard J, Frisvad JC. Terverticillate penicillia studied by direct electrospray mass spectrometric profiling of crude extracts.1. Chemo-systematics. Biochem Syst Ecol. 1997; 25: 51-64.

59. Li J, Tang G, Cheng K, Yang D, Chen G, Liu Z, et al. Peripheral blood mononuclear cell-based metabolomic profiling of a chronic unpredictable mild stress rat model of depression. Mol Biosyst. 2014; 10(11): 2994-3001.

60. Liu X, Zheng P, Zhao X, Zhang Y, Hu C, Li J, et al. Discovery and validation of plasma biomarkers for major depressive disorder classification based on liquid chromatography-mass spectrometry. J Proteome Res. 2015; 14(5): 2322-30.
61. Shao WH, Chen JJ, Fan SH, Lei Y, Xu HB, Zhou J, et al. Combined Metabolomics and Proteomics Analysis of Major Depression in an Animal Model: Perturbed Energy Metabolism in the Chronic Mild Stressed Rat Cerebellum. OMICS. 2015; 19(7): 383-92.

62. Wu H, Wang P, Liu M, Tang L, Fang J, Zhao Y, et al. A 1H-NMR-Based Metabonomic Study on the Anti-Depressive Effect of the Total Alkaloid of Corydalis Rhizoma. Molecules. 2015; 20(6): 10047-64.

63. Tian JS, Peng GJ, Gao XX, Zhou YZ, Xing J, Qin $\mathrm{XM}$, et al. Dynamic analysis of the endogenous metabolites in depressed patients treated with TCM formula Xiaoyaosan using urinary (1)H NMR-based metabolomics. J Ethnopharmacol. 2014; 158: 1-10.

64. Dickens AM, Larkin JR, Davis BG, Griffin JL, Claridge TD, Sibson NR, et al. NMR-Based Metabolomics Separates the Distinct Stages of Disease in a Chronic Relapsing Model of Multiple Sclerosis. J Neuroimmune Pharmacol. 2015; 10: 435-44.

65. Gonzalez-Dominguez R, Castilla-Quintero R, GarciaBarrera T, Gomez-Ariza JL. Development of a metabolomic approach based on urine samples and direct infusion mass spectrometry. Anal Biochem. 2014; 465: 20-27.

66. Sato Y, Nakamura T, Aoshima K, Oda Y. Quantitative and wide-ranging profiling of phospholipids in human plasma by two-dimensional liquid chromatography/mass spectrometry. Anal Chem. 2010; 82(23): 9858-64.

67. Dettmer K, Aronov PA, Hammock BD. Mass spectrometry-based metabolomics. Mass Spectrom Rev. 2007; 26(1): 51-78.

68. Katajamaa M, Oresic M. Data processing for mass spectrometry-based metabolomics. J Chromatogr A. 2007; 1158(1-2): 318-28.

69. Weaver PJ, Laures AM, Wolff JC. Investigation of the advanced functionalities of a hybrid quadrupole orthogonal acceleration time-of-flight mass spectrometer. Rapid Commun Mass Spectrom. 2007; 21(15): 2415-21.

70. Wang G, Zhou Y, Huang FJ, Tang HD, Xu XH, Liu $\mathrm{JJ}$, et al. Plasma metabolite profiles of Alzheimer's disease and mild cognitive impairment. J Proteome Res. 2014; 13(5): 2649-58.

71. Oreši M, Hyötyläinen T, Herukka SK, Sysi-Aho M, Mattila I, Seppänan-Laakso et al. Metabolome in progression to Alzheimer's disease. Transl Psychiatry. 2011; 1: e57.

72. Castrillo JI, Hayes A, Mohammed S, Gaskell SJ, Oliver SG. An optimized protocol for metabolome analysis in yeast using direct infusion electrospray mass spectrometry. Phytochemistry. 2003; 62: 929-37.

73. Cowen P.J. Cortisol, serotonin and depression: all stressed out?. Br.J.Psychiatry. 2002; 180: 99-100. 
74. Weng R, Shen S, Tian Y, Burton C, Xu X, Liu Y, et al. Metabolomics Approach Reveals Integrated Metabolic Network Associated with Serotonin Deficiency. Sci Rep. 2015; 5: 11864 .

75. Markus CR, Olivier B, Panhuysen GE, Van Der Gugten J, Alles MS, Tuiten A, et al. The bovine protein alpha-lactalbumin increases the plasma ratio of tryptophan to the other large neutral amino acids, and in vulnerable subjects raises brain serotonin activity, reduces cortisol concentration, and improves mood under stress. Am J Clin Nutr. 2000; 71(6): 1536-44.

76. Lanfumey L, Mongeau R, Cohen-Salmon C, Hamon M. Corticosteroid-serotonin interactions in the neurobiological mechanisms of stress-related disorders. Neurosci Biobehav Rev. 2008; 32(6): 1174-84.
* Corresponding author: Maria Luisa Bernal Ruiz Department of Pharmacology and Physiology Faculty of Medicine University of Zaragoza 50009 Zaragoza. Spain

Phone number: +34-976762602

Fax: +34-987761700

E-mail: mbernal@unizar.es 